\title{
Análise das condições bucal e mental de pessoas em situação de rua de Maringá-PR
}

\author{
Analysis of the oral and mental conditions of homeless people in Maringá-PR \\ Análisis de las condiciones orales y mentales de las personas sin hogar en Maringá-PR.
}

\begin{abstract}
RESUMO
Objetivo: Analisar os dados de saúde bucal e hábitos que colaboram para o agravamento da saúde bucal e mental dos moradores em situação de rua do município de Maringá-PR. Métodos: Pesquisa de caráter quantitativo/exploratório, realizada por meio da análise de prontuários eletrônicos da população em situação de rua (PSR). Os dados foram computados em uma planilha em Excel para análise estatística. Resultados: Foram quantificados e analisados 761prontuários eletrônicos. A faixa etária predominante foi a de 41,2 anos, e a predominância foi do sexo masculino. Os problemas bucais mais prevalentes foram relacionados à periodontia e cirurgia e com necessidades de próteses. Os dados também evidenciaram alto consumo de drogas ilícitas, álcool e tabaco, resultando numa alta prevalência de comorbidades e agravamento do quadro da saúde mental desta população. Conclusão: Espera-se que os resultados obtidos possam servir para embasar a política pública do município em relação a esta população especificamente, assim como avaliar a efetividade, ampliação e aprimoramento dos serviços do município de Maringá.
\end{abstract}

DESCRITORES: Pessoas em situação de rua; Políticas públicas de saúde; Promoção da saúde.

\section{ABSTRACT}

Goal: To analyze the oral health data and habits that contribute to the worsening of the oral and mental health of homeless residents in the city of Maringá-PR. Methods: Quantitative/exploratory research, carried out through the analysis of electronic medical records of the homeless popuIation (PSR). Data were computed in an Excel spreadsheet for statistical analysis. Results; It wasquantified and analyzed 761 electronic medical records. The predominant age group was 41.2 years, and the predominance was male. The most prevalent oral problems were related to periodontics and surgery and the need for prostheses. The data also showed a high consumption of illicit drugs, alcohol and tobacco, resulting in a high prevalence of comorbidities and a worsening of the mental health status of this population. Conclusion: is hoped that the results obtained may serve to support the municipality's public policy in relation to this population specifically, as well as to assess the effectiveness, expansion and improvement of services in the municipality of Maringá

DESCRIPTORS: Homeless people; Public health policies; Health promotion.

\section{RESUMEN}

Objetivo: Analizar los datos y hábitos de salud bucal que contribuyen al empeoramiento de la salud bucal y mental de los residentes en situación de calle en la ciudad de Maringá-PR. Métodos: Investigación cuantitativa / exploratoria, realizada a través del análisis de historias clínicas electrónicas de la población sin hogar (PSR). Los datos se calcularon en una hoja de cálculo de Excel para el análisis estadístico. Resultados: Cuantificamos y analizamos 761 historias clínicas electrónicas. El grupo de edad predominante fue de 41,2 años y el sexo masculino. Los problemas bucales más prevalentes se relacionaron con la periodoncia y la cirugía y la necesidad de prótesis. Los datos también mostraron un alto consumo de drogas ilícitas, alcohol y tabaco, lo que resultó en una alta prevalencia de comorbilidades y un empeoramiento del estado de salud mental de esta población. Conclusión: Se espera que los resultados obtenidos sirvan para apoyar la política pública del municipio en relación a esta población específicamente, así como para evaluar la efectividad, ampliación y mejora de los servicios en el municipio de Maringá.

DESCRIPTORES: Personas sin hogar. Políticas de salud pública. Promoción de la salud.

RECEBIDO EM: 08/06/21 APROVADO EM: 14/06/21

\section{Maria Paula Jacobucci Botelho}

Professora Doutora do Departamento de Odontologia da Universidade de Maringá - PR. UniCesumar. Odontóloga do SAE / CTA IST Aids de Maringá-PR.

ORCID: 0000-0003-0485-1307.

\section{Raul Sturion}

Acadêmico do Curso de Odontologia, Departamento de Odontologia - Universidade de Maringá-PR. UniCesumar. ORCID: 0000-0002-2999-6070 


\section{Janus Micael Targa Ferreira}

Acadêmico do Curso de Odontologia na UniCesumar, bolsista PIBIC/UniCesumar. ORCID: 0000-0003-3138-2200

\section{Michel Rangel}

Odontólogo da Estratégia Saúde da Família. Coordenador de Saúde Bucal da Secretaria Municipal de Saúde de Maringá-PR. ORCID: 0000-0002-7370-4020

\section{Isabela Miguel Pissioli}

Médica da Equipe do Consultório na Rua.

ORCID: 0000-0002-6305-7224

\section{Maria Heloísa Cella}

Psicóloga. Gerente de Saúde Mental, Secretaria Municipal de Maringá-PR.

\section{INTRODUÇÃO}

As desigualdades sociais estabelecidas pelo sistema capitalista levaram as sociedades modernas a marginalizar determinados grupos sociais. Ao longo do tempo pode ser notado um crescente aumento da População em Situação de Rua (PSR), que é definida pela Política Nacional para a População em Situação de Rua, e de acordo com o Ministério de Desenvolvimento Social e Combate à Fome como um grupo populacional heterogêneo, composto por pessoas com diferentes realidades, mas que têm em comum a condição de pobreza absoluta, vínculos interrompidos ou fragilizados e inexistência de moradia convencional regular, sendo compelidos a utilizarem a rua como espaço de moradia e sustento, por contingência temporária ou de forma permanente1.

Diversas são as situações que podem conduzir os indivíduos às ruas. Além de fatores socioeconômicos, como a pobreza, podemos destacar também os fatores relacionados aos convívios interpessoais, como problemas familiares ligados à rejeição do indivíduo pela família, atitudes violentas e hostilidades. A PSR é constituída em grande parte por pessoas ignoradas do convívio social e, devido à sua condição de inutilidade social, se tornam invisíveis e passam a não ser notadas pela sociedade. Portanto, é necessário o reconhecimento desse grupo na sociedade como detentores de direito e com livre acesso à saúde2, mas isto não ocorre.

Dentre as políticas públicas de saúde oferecidas à PSR destaca-se o Consultório na Rua (CnaR), que foi instituída através da Política Nacional de Atenção Básica (PNAB) e que consiste em um consultório móvel, oferecendo um diferencial da estrutura física tradicional de consultório. O CnaR é composto por uma equipe multidisciplinar que faz busca ativa nos locais onde se encontra a PSR, desta forma fazendo o acolhimento e a formação de vínculos entre profissionais de saúde, assistentes sociais e a PSR, melhorando o acesso e o direito à saúde, visando sanar suas necessidades3.

As principais atividades e ações são desenvolvidas na rua e articuladas com os demais serviços e centros de saúde, como a Unidade Básica de Saúde (UBS), serviços hospitalares de Urgência e Emergência, Centros de Atenção Psicossocial (CAPS), Unidades de Acolhimento, Centros de Saúde, Centros Populares, entre outros4.

Também é importante compreender e refletir sobre o cuidado às pessoas em situação de rua, em especial aquelas que apresentam transtornos mentais. Quando se encontram em situação de rua há exacerbação de seus sintomas, além de acúmulo de vários outros distúrbios, resultando em alta prevalência de comorbidades e agravamento do quadro de saúde mental. Nesta população o transtorno mental mais comum está associado à dependência de álcool e outras drogas, variando entre $8 \%$ e $58 \%$ para a dependência de álcool e $5 \%$ a $54 \%$ para outras drogas5.

Em Maringá a equipe atualmente é de modalidade III, conta com um médico, enfermeiro, técnico de enfermagem, psicólogo, assistente social e um motorista. A equipe dispóe de um carro adaptado para atendimento in loco da PSR e há um número de telefone celular para o contato com a equipe, que realiza todos os procedimentos da carteira de serviços do SUS e compartilha o cuidado com a rede de atenção do município.

Viabilizar atendimentos de saúde para PSR é proporcionar equidade a todos os cidadãos, direito este que garante igualdade e justiça, reconhece as necessidades específicas de cada grupo social e reduz o impacto dos determinantes sociais da saúde aos quais esse grupo é submetido6. Sendo assim, o objetivo geral da pesquisa foi analisar a saúde bucal da PSR, os hábitos que colaboram para o agravamento dos problemas bucais e transtornos mentais das pessoas em situação de rua do município de Maringá.

\section{MÉTODOS}

O projeto está vinculado ao curso de Odontologia da UniCesumar (Maringá-PR) e foi aprovado por seu Comitê de Ética (Parecer TCLE/CAAE no 3.702.180). Foi desenvolvido de acordo com os pressupostos, as diretrizes e as normas regulamentadoras da pesquisa envolvendo seres humanos. Também foi submetido ao Comitê de Ética em Pesquisa da Secretaria Municipal de Saúde de Maringá e ao Centro de Capacitação e Pesquisas em Projetos Sociais, sendo autorizada pela Comissão Permanente de Avaliação de Projetos - Portaria $n^{\circ}$ 029/2019-SAÚDE a ser realizada na Se- 
cretaria de Saúde de Maringá: Consultório na Rua no período de 2019 a 2021.

$\mathrm{O}$ estudo consistiu em uma pesquisa de caráter quantitativo e exploratório para a análise das condições de saúde da PSR. O estudo incluiu a análise de prontuários eletrônicos da PSR do município de Maringá, que passaram por consultas nos anos de 2019 e 2020. Essa coleta de dados foi realizada junto à Secretaria de Saúde de Maringá. A segunda etapa fez a caracterização da população em situação de rua em Maringá, através dos dados coletados e de entrevistas com pessoas que vivem em situação de rua e que aceitaram participar do estudo após serem informados a respeito dos objetivos e propósitos da pesquisa. Após serem informados, foi solicitado que assinassem o Termo de Consentimento Livre e Esclarecido - TCLE.

No entanto, a segunda parte da pesquisa não foi desenvolvida em sua totalidade desta forma devido à calamidade pública provocada pela pandemia da COVID-197 e as restrições advindas dela. Os dados foram coletados no período anterior a esses decretos.

Os dados obtidos foram digitados em planilha do programa Microsoft Excel 2010 e analisados estatisticamente com o auxílio do Software Statistica Single User versão 13.2 no ano de 2021. Foram calculadas medidas descritivas: média, desvio padrão, mínimo e máximo para as variáveis quantitativas e tabelas simples e de dupla entrada para as variáveis qualitativas também em 2021. Para verificar possíveis associações entre as variáveis qualitativas, foi realizado o teste qui-quadrado, com nível de significância de $5 \%$, ou seja, foram consideradas significativas as comparações cujo $\mathrm{p}<0,05$.

\section{RESULTADOS}

Dos 761 prontuários analisados, 87\% eram homens, com idade entre 30 a 49 anos $(59,7 \%)$. Somente $10 \%$ passaram por atendimentos odontológicos $(\mathrm{n}=77)$ sendo que a maioria procedimentos cirúrgicos. $28,8 \%$ $(\mathrm{n}=219)$ utilizavam algum tipo de droga, $25,8 \%$ eram tabagistas $(n=196)$ e $29,9 \%$ ( $\mathrm{n}=205)$ consumiam bebida alcoólica.(ta-
Tabela 1. Distribuição das variáveis avaliadas nos entrevistados

\begin{tabular}{|c|c|c|c|c|c|c|}
\hline & Variáveis & $\mathrm{N}$ & $M$ & $F$ & $\%$ & $p$ \\
\hline \multirow[t]{3}{*}{ Tabagismo } & Sim & 196 & 169 & 27 & 25,8 & 0,7366 \\
\hline & Não informou & 492 & 430 & 62 & 64,7 & \\
\hline & Não tabagista & 17 & 16 & 1 & 2,2 & \\
\hline \multirow[t]{3}{*}{ Etilismo } & Sim & 205 & 174 & 26 & 26,9 & \\
\hline & Sem informação & 465 & 407 & 16 & 61,1 & 0,1897 \\
\hline & Não consome & 20 & 19 & 1 & 2,6 & \\
\hline \multirow[t]{3}{*}{ Cocaína } & Sim & 41 & 35 & 6 & 5,4 & 0,1422 \\
\hline & Não & 212 & 178 & 34 & 27,9 & \\
\hline & Não informou & 508 & 453 & 55 & 66,8 & \\
\hline \multirow[t]{3}{*}{ Crack } & Sim & 81 & 63 & 18 & 10,6 & $0,0156^{*}$ \\
\hline & Não & 172 & 150 & 22 & 22,6 & \\
\hline & Não informou & 508 & 453 & 55 & 66,8 & \\
\hline \multirow[t]{3}{*}{ Maconha } & Sim & 53 & 42 & 11 & 7,0 & 0,0693 \\
\hline & Não & 200 & 171 & 29 & 26,3 & \\
\hline & Não informou & 508 & 453 & 55 & 66,8 & \\
\hline \multirow[t]{3}{*}{ Outras } & Não & 189 & 159 & 30 & 24,8 & 0,1465 \\
\hline & Sim & 64 & 10 & 54 & 8,4 & \\
\hline & Não informou & 508 & 453 & 55 & 66,8 & \\
\hline \multirow[t]{3}{*}{ Drogas ilícitas } & Sim & 219 & 180 & 39 & 28,8 & $0,0075^{*}$ \\
\hline & Não & 1 & 0,1 & 33 & 4,3 & \\
\hline & Não informou & 55 & 7,2 & 453 & 59,5 & \\
\hline
\end{tabular}

Tabela 2. Distribuição das variáveis avaliadas nos entrevistados

$\begin{array}{lccccccc} & & \text { N } & \text { M } & \text { F } & \% & \text { P }^{*} \\ \text { DCNT } & \text { Sim } & 160 & 134 & 26 & 21,0 & 0,2681 \\ & \text { Não } & 18 & 16 & 2 & 2,4 & \\ \text { DI } & \text { Sem informação } & 583 & 516 & 67 & 76,6 & \\ & \text { Sim } & 67 & 53 & 14 & 8,8 & 0,0789 \\ & \text { Não } & 25 & 26 & 2 & 3,3 & \\ \text { Desmaios/con- } & \text { Sem informação } & 669 & 590 & 79 & 87,9 & \\ \text { vulsões } & \text { Sim } & 6 & 6 & 0 & 0,8 & 0,5293 \\ & \text { Não } & 24 & 22 & 2 & 3,2 & \\ \text { Utilização de } & \text { Sem informação } & 731 & 638 & 93 & 96,1 & \\ \text { medicamentos } & \text { Sim } & 148 & 121 & 27 & 19,4 & 0,0617 \\ & \text { Não } & 35 & 30 & 5 & 4,6 & \\ \text { Cárie } & \text { Sem informação } & 578 & 515 & 63 & 76,0 & \\ & \text { Sim } & 69 & 58 & 11 & 9,1 & 0,3621 \\ \text { *Testequi-quadradonão significativo considerando nível de significânciade } 5 \% & & & & \end{array}$


bela1)

Em relação a outras condições clínicas com registro nos prontuários eletrônicos dos pacientes, 21\% $(\mathrm{n}=160)$ apresentam outras doenças que não Doenças Crônicas Não Transmissíveis (DCNT) e alergias; $8,8 \%(\mathrm{n}=67)$ apresentam doenças infecciosas (DI), 3,2\% $(n=24)$ apresentaram desmaios e convulsões, $11,8 \%(\mathrm{n}=90), 19,4 \%$ faziam uso de medicamentos $(n=148)$ $1,1 \%$. Já em relação à presença de doenças e utilização de medicações, não houve diferenças estatisticamente significativas entre os grupos. Podemos considerar a proporção de dados discrepantes devido à dificuldade para serem obtidos os dados em relação a esse parâmetro.(tabela 2)

Já em relação à presença de doenças e utilização de medicações, não houve diferenças estatisticamente significativas entre os grupos.

\section{DISCUSSÃO}

A ausência de muitos dados nos prontuários eletrônicos da Secretaria de Saúde de Maringá-PR reforçam a invisibilidade da PSR, além da dificuldade que encontram para o acesso aos mais diversos tratamentos em Maringá, como outros estudos já haviam pontuado8. O não comprometimento no atendimento a essa população também faz com que as informações não sejam coletadas adequadamente e isso pode agravar o sentimento de não pertencimento dessa população, acentuando problemas de saúde mental como foi observado neste estudo. $\mathrm{Na}$ área da saúde o objetivo é a produção do cuidado e, assim, será possível alcançar a cura e a saúde. Há múltiplas ma- neiras, modelos e ações para fazer o cuidado. $\mathrm{O}$ modelo biomédico já mostrou não ser a melhor forma para fazer o cuidado. É preciso trabalhar sob o paradigma psicossocial, com equipe interprofissional, com diferentes recursos terapêuticos, enfatizando a reinserção social dos indivíduos9. A PSR precisa de mais visibilidade, atenção e novas políticas devem ser instituídas para poder oferecer um atendimento mais adequado.

Há necessidade de implementar os atendimentos odontológicos pela rede para mudar essa realidade, além de incorporar um profissional de saúde bucal no CnaR trabalhando em conjunto nas abordagens da PRS para identificar suas necessidades e saná-las precocemente10. Apesar de a PSR ter baixas condições de saúde bucal, sistêmica e mental tem merecido pouca atenção por parte da comunidade científica, com poucos estudos publicados a respeitol1. Contribui para essas más condições de saúde bucal, o alto consumo de drogas, álcool e tabaco pela PSR. Esse consumo pode ser explicado pela necessidade dessa população de minimizar a fome e o frio, como também para a socialização entre os membros dos grupos da rua12.

Ao utilizar drogas, particularmente o crack, ocorre o manchamento dos dentes, comprometendo ainda mais a autoestima das pessoas. Assim, a realização de procedimentos restauradores com finalidade estética também é importante no resgaste da autoestima e permite melhor reintegração na sociedade. Saúde bucal está relacionada à saúde sistêmica e mental e deve ser considerada fundamental no atendimento à PSR. Da mesma forma, problemas de saúde mental representam a razão predominante de essas pessoas estarem em situação de rua e devem ser considerados sempre. A ampliação do atendimento em saúde para a PSR é necessária, mas só ocorrerá quando houver maior visibilidade e preocupação com essa população.

Dentre os fatores limitantes para a realização desta pesquisa, cabe destacar a falta de informações precisas de uma parcela de pacientes e a pandemia. A não existência de informações destaca a invisibilidade desta população. Estes resultados reforçam a importância de aprimorar a atenção à saúde da PSR e proporcionar soluções às necessidades de saúde bucal percebidas por esse grupo invisível à sociedade, contribuindo para uma melhor qualidade de vida e o restabelecimento da sua dignidade.

\section{CONCLUSÃO}

Este estudo comprovou que falta acolhimento, escuta ampliada, empatia no atendimento a essa população e, com isso, problemas mentais vão se agravando. É preciso maior comprometimento por parte do poder público e dos profissionais da saúde no cuidado a esses pacientes. É evidente que a PSR possui baixas condições de saúde, como resultado das condições precárias em que vivem. Isto não é novidade. O que é preciso após essa constatação é atuar de forma mais efetiva para mudar essa realidade.

Os resultados alcançados reforçam a importância de aprimorar a atenção à saúde da PSR e proporcionar soluções às necessidades de saúde percebidas, contribuindo para uma melhor qualidade de vida e o restabelecimento da sua dignidade, com sua reinserção na sociedade.

\section{REFERÊNCIAS}

1. Brasil. I Encontro Nacional sobre População em Situação de Rua: relatório. Brasília, DF: Ministério do Desenvolvimento Social e Combate à Fome, Secretaria de Avaliação e Gestão da Informação, Secretaria Nacional de Assistência Social, 2006a.

2. Silva LMA, Monteiro IS, Araújo ABVL. Saúde bucal e consultório na rua: o acesso como questão central da discussão. Cadernos Saúde Coletiva, 2018;26(3):285-91.

3. Londero MFP, Ceccim RB, Bilibio LFS. Consultório de/na rua: desafio para um cuidado em verso na saúde. Interface-Comunicação,
Saúde, Educação, 2014;18:251-60.

4. Brasil. Ministério da Saúde. Portaria n. 2.436, de 21 de setembro de 2017. Aprova a Política Nacional de Atenção Básica, estabelecendo a revisão de diretrizes para a organização da Atenção Básica, no âmbito do Sistema Único de Saúde (SUS). Diário Oficial da União, 2017;183:1.

5. Fazel S, Geddes J R, Kushel M. The health of homeless people in highincome countries: descriptive epidemiology, health consequences, and clinical and policy recommendations. Lancet, 2014: 


\section{REFERÊNCIAS}

384(9953), 1529-40

6. Brasil. Ministério da Saúde. Manual sobre o cuidado à saúde junto a população em situação de rua. Brasília: Ministério da Saúde, 2012.

7. Brasil. Decreto Legislativo n. ${ }^{\circ}$ 6, de 2020. Reconhece o estado de calamidade pública. Diário Oficial da União. 2020 mar 18;55-C:1:col.1. [Citado em 17 Ago 2020]. Disponível em: http:// www.planalto.gov.br/ccivil_03/portaria/DLG6-2020.htm

8. Engstrom EM, Teixeira MB. Equipe "Consultório na Rua" de Manguinhos, Rio de Janeiro, Brasil: práticas de cuidado e promoção da saúde em um território vulnerável. Ciência \& Saúde Coletiva 2016;21:1839-1848,2016.

9. Silva LVC, Muniz MPG, Caçador BS, Caram CS, Brito MJM. Práticas de cuidado em saúde mental com população privada de liberdade: revisão de escopo. Saúde Coletiva 2021;11(69):8236-8241.

10. Couto JGA. Atenção à Saúde Bucal da População em Situação de Rua: uma análise das ações nas capitais brasileiras da região sul. 2016.

11. Lawder JAC, Matos MAD, Souza JBD, Freire MDCM. Impact of oral condition on the quality of life of homeless people. Revista de Saúde Pública, 2019;53:22.

12. Botti NCL, Castro CGD, Silva MFD, Silva AK, Oliveira LCD, Castro ACHOAD, Fonseca LLK. Prevalência de depressão entre homens adultos em situação de rua em Belo Horizonte. J Bras Psiquiatr, 2010:59(1):10-6. 